\title{
Development of Single-Walled Carbon Nanotube-Based Biosensor for the Detection of Staphylococcus aureus
}

\author{
Hyun-Kyung Choi, ${ }^{1}$ Jinyoung Lee, ${ }^{1}$ Mi-Kyung Park, ${ }^{2}$ and Jun-Hyun Oh ${ }^{1}$ \\ ${ }^{1}$ Department of Plant and Food Sciences, Sangmyung University, 31 Sangmyungdae-gil, Dongnam-gu, Cheonan, \\ Chungnam 31066, Republic of Korea \\ ${ }^{2}$ School of Food Science and Biotechnology, Kyungpook National University, 80 Daehak-Ro, Buk-Gu, Daegu 41566, Republic of Korea
}

Correspondence should be addressed to Mi-Kyung Park; parkmik@knu.ac.kr and Jun-Hyun Oh; junhyunoh@smu.ac.kr

Received 16 March 2017; Revised 16 September 2017; Accepted 8 October 2017; Published 23 November 2017

Academic Editor: Jorge Barros-Velázquez

Copyright (C) 2017 Hyun-Kyung Choi et al. This is an open access article distributed under the Creative Commons Attribution License, which permits unrestricted use, distribution, and reproduction in any medium, provided the original work is properly cited.

\begin{abstract}
The goal of this research is to develop a single-walled carbon nanotube- (SWCNT-) based biosensor to detect Staphylococcus aureus. The specificity of 11 bacteria and polyclonal anti-Staphylococcus aureus antibodies (pAbs) was determined using an indirect ELISA. The pAbs were immobilized onto sensor platform after the hybridization of 1-pyrenebutanoic acid succinimidyl ester (PBASE). The resistance difference $(\Delta R)$ was calculated using a potentiostat. The bacteria detected by the biosensor were observed using a scanning electron microscope (SEM). The optimum concentration of SWCNTs on the platform was determined to be $0.1 \mathrm{mg} / \mathrm{mL}$. The binding of pAbs with $S$. aureus resulted in a significant increase in resistance value of the biosensor $(P<0.05)$. The SEM images confirmed the specific binding of $S$. aureus on the biosensor. The SWCNT-based biosensor was able to detect $S$. aureus with a limit of detection (LOD) of $4 \log \mathrm{CFU} / \mathrm{mL}$.
\end{abstract}

\section{Introduction}

Processed foods are monitored worldwide to protect people from infectious diseases primarily caused by foodborne pathogenic bacteria. Among the foodborne disease-causing bacteria involved in predominant foodborne disease outbreaks, Staphylococcus aureus is one of the leading causes of gastroenteritis resulting from the consumption of contaminated food [1]. Since 2010 in South Korea, approximately 1,180 people have been hospitalized from 66 outbreaks of Staphylococcus [2]. Staphylococcal food poisoning is due to the absorption of staphylococcal enterotoxins preformed in the food [3]. The major symptoms of staphylococcal enteritis include nausea, vomiting, severe abdominal cramps, diarrhea, sweating, and headache. Signs of toxicity, such as fever and hypotension, are rarely observed in cases of staphylococcal food poisoning.

Traditional detection methods for microorganisms such as plate counting agar (PCA) are arduous and time-consuming methods, requiring more than $24 \mathrm{~h}$. In addition, it has been recognized that the culturing-based methods can underestimate the pathogen numbers due to the limitations associated with culturing such as viable but nonculturable cells [4]. Polymerase chain reaction (PCR), quantitative real-time PCR (qPCR), and enzyme-linked immunosorbent assay (ELISA) methods have improved both the speed and sensitivity of pathogen detection, compared with detection by the traditional culturing methods $[5,6]$. However, PCR techniques often have high risk of false results owing to inhibition by components of the sample matrix and complicated pretreatment process such as extraction of the whole or a part of the pathogen DNA [5]. ELISA technique also requires extra-labeled antibodies that are costly and time-consuming steps [7].

Recently, nanowires, nanotubes, and nanospheres as donors of electrical responses have been studied for minimized nanostructures in a field of biosensors. Nanoscaled biosensor devices can support in vivo applications, high sensitivity, and low limit concentration of detection [8]. In addition, a number of researchers conducting a study for nanoscaled biosensors are trying to develop easy detection methods that are label-free, rapid, low-cost, and involving 
multiplexed analysis. Single-walled carbon nanotubes (SWCNTs) are attractive materials to be used for nanoelectronics [9-11]. The electrical properties of SWCNTs are especially good for uses in advanced biological electronics and biosensors. SWCNTs and gold assembly onto silicon wafers enable researchers to affect the electrical response for high sensitivity of the biosensor.

There are several methods to immobilize the biomolecules onto SWCNTs. The covalent bonding methods onto SWCNTs using modification with chemical functional groups may cause problems concerning the electrical properties of SWCNTs $[12,13]$. On the contrary, noncovalent bonding method using $\pi-\pi$ stacking does not enable the transferring of chemical characteristics because it only utilizes physical forces to immobilize materials onto SWCNTs. Enzyme immobilization is also one of the most important processes for increasing the sensitivity and stability of the biosensor. The low activity of the immobilized enzyme is due to different local $\mathrm{pH}$ or electrostatic interactions at the matrix-enzyme interface, covalent linking to enzyme changing overall enzyme structure, and matrix induced confinement decreasing enzyme mobility for conformation changes during substrate catalysis [14-16].

In food industry, biosensor methods are receiving a great amount of attention due to the potential feasibility of practical on-site detection of foodborne pathogens as rapid detection methods. In addition, biosensor methods do not require a high level of operator expertise and complicated sample preparation steps [17]. The overall goal of this research is to develop and characterize a SWCNT-based biosensor to detect Staphylococcus aureus. The specific objectives of this research are to (1) investigate the binding specificity between the target Staphylococcus aureus and polyclonal anti-Staphylococcus aureus antibodies for biosensor applications; (2) develop a fabrication process for SWCNT-based biosensor; and (3) characterize the properties of SWCNT-based biosensor to detect Staphylococcus aureus.

\section{Materials and Methods}

2.1. Materials. A stock culture of Staphylococcus aureus KCTC 3881 was purchased from the Korea Research Institute Bioscience and Biotechnology (Daejeon, South Korea). The culture of $S$. aureus was maintained in nutrient broth (Difco Laboratories, Detroit, MI, USA) by incubating for $16 \mathrm{~h}$ at $37^{\circ} \mathrm{C}$. The other bacteria including Yersinia enterocolitica ATCC23715, Salmonella typhimurium ATCC19586, Klebsiella pneumoniae ATCC13883, E. coli O157:H7, Salmonella enteritidis ATCC19586, Shigella boydii ATCC11190, Pseudomonas aeruginosa ATCC10145, Listeria monocytogenes ATCC19116, Bacillus cereus ATCC21768, and Shigella sonnei ATCC9290 were obtained from the Food Safety Laboratory at Kyungpook National University (Daegu, South Korea). The singlewalled carbon nanotubes (SWCNTs) were purchased from Chengdu Organic Chemicals Co. Ltd. (Chengdu, China) and the purity was greater than 95\%. N,N-Dimethylformamide (DMF) was purchased from Daejung Inc. (Siheung, South Korea). 1-Pyrenebutanoic acid, succinimidyl ester (PBASE) was purchased from Thermo Fisher Scientific Inc. (Waltham,
MA, USA). A polyclonal anti-Staphylococcus aureus antibody (pAb) was purchased from Abcam Inc. (Cambridge, UK) and diluted to a concentration of $4 \mathrm{mg} / \mathrm{mL}$ with carbonatebicarbonate buffer prior to use. Other reagents used in this research were of analytical grade.

2.2. Indirect ELISA for Binding Specificity of pAbs with Bacteria. The specificity of pAbs was compared with commercially available anti-staphylococcus aureus using an indirect ELISA following the procedures described in the previous study [18]. S. aureus was cultivated in nutrient broth (Difco Laboratories, Detroit, MI, USA), and the other bacteria were cultivated in tryptic soy broth (Difco Laboratories, Detroit, MI, USA) in a shaking incubator at $37^{\circ} \mathrm{C}$ for $16 \mathrm{~h}$. The grown and collected bacteria were washed three times with $10 \mathrm{mM}$ PBS buffer (pH 7.4) by centrifugation at $5,000 \mathrm{rpm}$ for $1 \mathrm{~min}$ at $4^{\circ} \mathrm{C}$. An aliquot of $100 \mu \mathrm{L}$ anti-Staphylococcus aureus antibodies diluted ten thousandfold with PBS buffer was taken and incubated in each well of the microplate (SPL, Pocheon, South Korea). Then, the color development was measured using a microplate reader (Synergy H1 hybrid reader, Seoul, South Korea) at $405 \mathrm{~nm}$. The result was calculated using the following equation [19]:

$$
\begin{aligned}
& \text { Difference of absorbance } \\
& \begin{aligned}
= & \text { Absorbance at } 405 \mathrm{~nm} \text { after } 30 \mathrm{~min} \\
& - \text { Absorbance at } 405 \mathrm{~mm} \text { at } 0 \mathrm{~min} .
\end{aligned}
\end{aligned}
$$

2.3. Fabrication of Mask and Sensor Platform. A mask for the sensor platform was fabricated prior to manufacturing the biosensor platform. The pattern of gold deposition as electrodes on the platform was designed to achieve a maximum of six measurements at one-time treatment (Figure 1(a)). The distance between each gold deposition was approximately $0.1 \mathrm{~cm}$. According to the mask designed, gold was deposited on the surface of a $\mathrm{Cr}$-deposited silicon wafer using an electron-beam evaporator (SRN-110-1505-R2, Sorona Inc., Pyeongtaek, South Korea) under vacuum at $4.0 \times 10^{-6}$ torr. The conductivity of Cr-deposited silicon wafer was tested before gold deposition to ensure that there was no electrical current on the surface. The conductivity of gold-deposited platform was also tested to confirm that the gold electrodes were working properly when the electrodes were connected by the electrical tester.

2.4. Optimum Concentration of SWCNTs on the Sensor Platform. The SWCNT powder was suspended in DMF solution by sonicating for $120 \mathrm{~min}$ in a water-filled sonicator (UCP02, Jeiotech, Daejeon, South Korea) at room temperature. The assembly of SWCNTs on the sensor platform was performed by drop-casting $5 \mu \mathrm{L}$ SWCNT solution onto the center of the surface of the gold-deposited biosensor platform to cover each space between the gold electrodes. The platform was then incubated for $15 \mathrm{~min}$ at $80^{\circ} \mathrm{C}$ in a drying oven to remove DMF residue and allowed to align SWCNTs between the gold electrodes. The resistance value $(\mathrm{k} \Omega)$ of the SWCNT bundle plate was measured using a potentiostat (DY2013, EG Technology, Seoul, South Korea) to confirm the assembly 


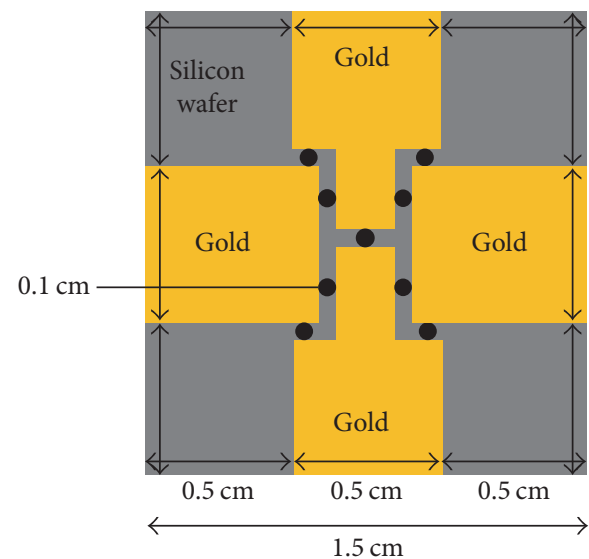

(a)

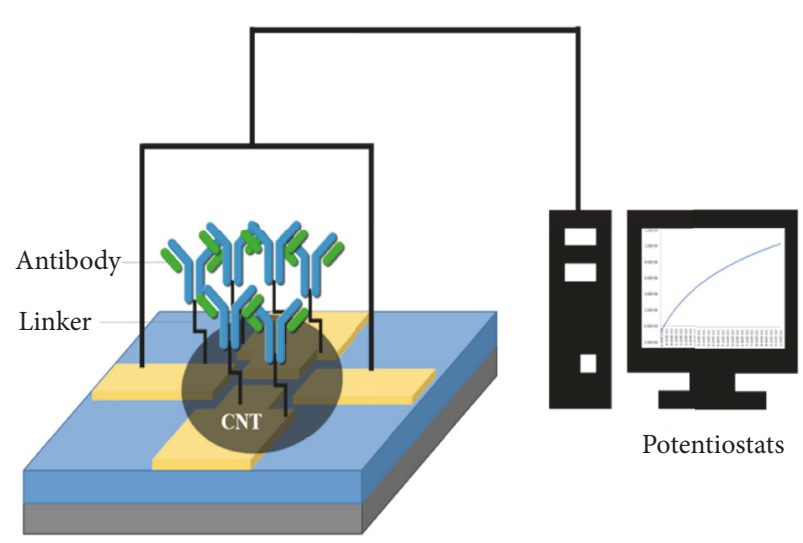

(b)

FIGURE 1: Platform design (a) and diagram of SWCNT-based biosensor (b).

of SWCNTs between the gold electrodes. The optimum concentration of SWCNTs was determined by applying the selected SWCNT-concentrations of $0.02,0.1,1$, and $10 \mathrm{mg} / \mathrm{mL}$ onto the biosensor platform and washing the surface of the platform with $1 \mathrm{~mL}$ deionized (DI) water up to five times. The resistance value was measured after each washing step.

2.5. Fabrication of SWCNT-Based Biosensor. For the noncovalent functionalization of the SWCNT bundles, the biosensor platform assembled with SWCNTs was further incubated with $6 \mathrm{mM}$ PBASE (9.8 $\mathrm{mg}$ PBASE in $5 \mathrm{~mL}$ DMF) as a linker for $2 \mathrm{~h}$ at room temperature followed by rinsing with pure DMF and DI water to remove excessive PBASE on the biosensor [20]. An anti-Staphylococcus aureus antibody at a concentration of $4 \mathrm{mg} / \mathrm{mL}$ was centrifuged at $12,000 \mathrm{rpm}$ for $20 \mathrm{~s}$ and diluted ten thousandfold with carbonate-bicarbonate buffer ( $\mathrm{pH}$ 9.3). The antibody was immobilized on the surface of SWCNT bundles by exposing the antibody to the PBASE linker overnight at $4^{\circ} \mathrm{C}$, allowing the formation of covalent bonds with PBASE on the SWCNT bundles. The SWCNT-based biosensor immobilized with pAbs was then rinsed with PBS buffer ( $\mathrm{pH}$ 7.4). Figure 1(b) illustrates the basic structure of the SWCNT-based biosensor immobilized with antibody. The resistance value $(\mathrm{k} \Omega)$ of the antibodyimmobilized biosensor was measured using a potentiostat (DY2013, EG Technology, Seoul, South Korea). The resistance measurements were conducted each step during the fabrication of SWCNT-based biosensor.

2.6. Detection of S. aureus Using an SWCNT-Based Biosensor. $S$. aureus cells were collected from the fully grown culture after washing 3 times with $10 \mathrm{mM}$ PBS (pH 7.4) using a centrifuge at $5000 \mathrm{rpm}$ for $5 \mathrm{~min}$ at $4^{\circ} \mathrm{C}$. The collected bacterial populations were estimated by referring the optical density (OD) measured at $650 \mathrm{~nm}$ using a spectrophotometer (Uvmini 1240, Shimadzu, Kyoto, Japan) to a preconstructed standard curve for $\log \mathrm{CFU} / \mathrm{mL}$.

Aliquots of $100 \mu \mathrm{L}$ stock solution of $S$. aureus culture were dropped onto the SWCNT-based biosensor and incubated for $30 \mathrm{~min}$ at room temperature to allow specific bindings between the bacteria and antibody. The control biosensor was prepared by applying $100 \mu \mathrm{L}$ of PBS buffer on the SWCNTbased biosensor immobilized with antibody instead of $S$. aureus culture. Linear sweep voltammetry was measured after incubation using a potentiostat (DY2013, EG Technology, Seoul, South Korea). The slopes of the current/voltage $(I / V)$ curves between 0 and $0.1 \mathrm{~V}$ for each treatment were calculated using linear regression analysis with the resistance $(R)$ values calculated by inversing the current/voltage value [21]. The resistance difference $(\Delta R)$ was then calculated using the following equation:

$$
\Delta R=\frac{\left(R_{1}-R_{0}\right)}{R_{0}},
$$

where $R_{0}$ is the resistance measured with linker using the biosensor and $R_{1}$ is the resistance measured with Staphylococcus aureus culture using the biosensor.

2.7. Scanning Electron Microscopy. The microstructures of the surface of the control and the S. aureus-detected SWCNTbased biosensor were observed using a scanning electron microscope (SEM) (SNE-4500M, SEC Cooperation, Suwon, South Korea). The biosensor specimens were coated with gold using a sputter-coater (MCM-100, SEC Cooperation, Suwon, South Korea) prior to SEM observation. The surfaces of the gold-coated sensor platforms were examined with an SEM under an electric voltage of $30 \mathrm{kV}$ at a working distance of $3 \mathrm{~mm}$ with a $30,000 \mathrm{x}$ magnification.

2.8. Statistical Analysis. The potentiostat measurements were repeated six times. One-way analysis of variance (ANOVA) of the measurement was performed using SPSS software (version 11.5, SPSS Ins., Chicago, IL, USA) and the differences among means were analyzed using Duncan with the defined significance level of $P<0.05$.

\section{Results and Discussion}

3.1. Binding Specificity of pAbs with Bacteria. Indirect ELISA was performed to demonstrate the specific binding of tested 


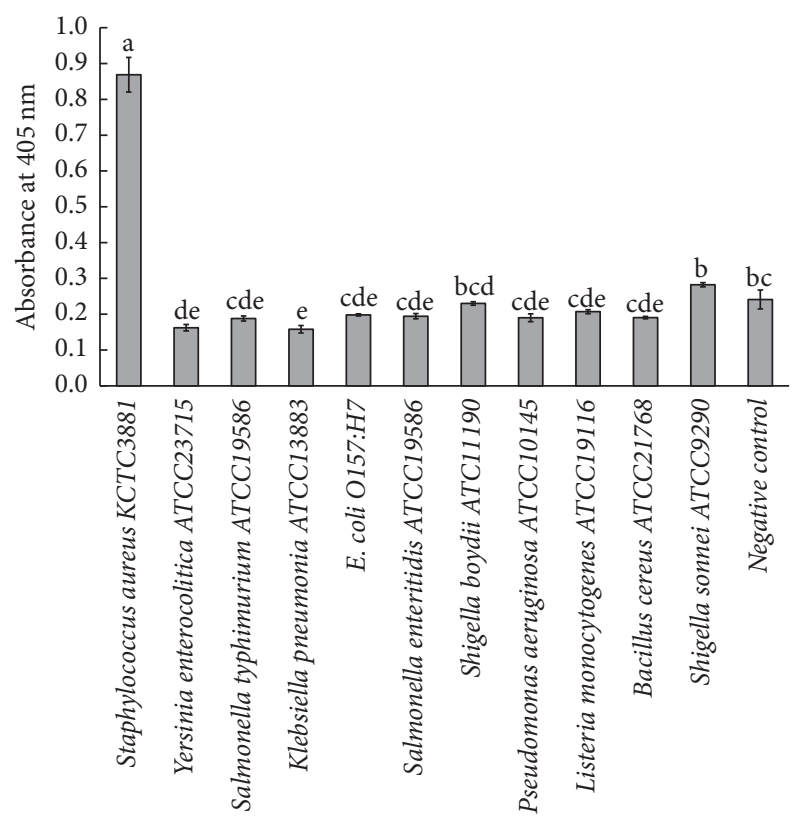

FIGURE 2: The specificity of custom prepared anti-Staphylococcus aureus polyclonal antibodies with selected bacterial strains determined by indirect ELISA. Error bars represent standard deviation $(n=3)$. Different superscript letters (a-e) represent significant differences among the samples at $P<0.05$.

S. aureus with pAbs. The indirect ELISA method involved the primary antibodies without enzyme and the secondary antibodies (which reacted with primary antibodies) combined with enzymes. When the enzymes react with substrates that can produce a fluorescent compound, color reaction occurs between the substrates and the enzymes combined with secondary antibodies [18]. The concentration of the tested bacterial species was approximately $1 \times 10^{8} \mathrm{CFU} / \mathrm{mL}$.

When the pAbs were diluted ten thousandfold and tested on the biosensor, the resistance of the biosensor was increased significantly from the preliminary experiment. Therefore, the ten thousand fold-dilution of pAbs was used for indirect ELISA. The specificity of the pAbs for the 11 tested bacteria was presented in Figure 2. The pAbs exhibited sufficient specificity with Staphylococcus aureus strains only, while the pAbs did not exhibit any specificity with other tested bacteria, evident from the absorbance values less than the cutoff value of 0.249 (mean of negative control +0.008 ). Based on the specific binding of pAbs to $S$. aureus only confirmed by indirect ELISA, the pAbs were demonstrated as the suitable antibody for the development of biosensor to detect $S$. aureus.

\subsection{Optimum Concentration of SWCNTs on Sensor Platform.} In general, CNT possesses a great potential for the applications to solid-state devices due to its small size as well as its superior electronic properties [22]. The immobilization of SWCNTs onto the sensor platform was conducted and confirmed by determining the resistance values at the selected concentrations of SWCNTs (Table 1). As the immobilized SWCNT-concentrations increased, the resistance values of

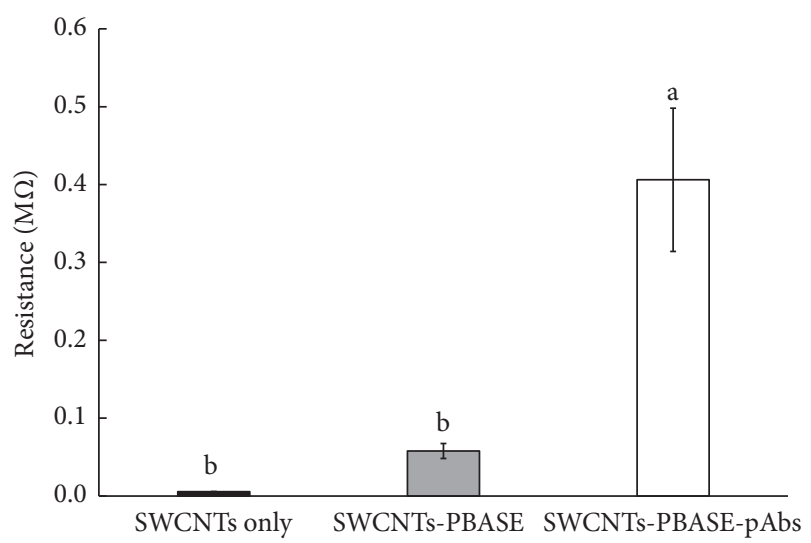

FIGURE 3: Resistance after immobilization of SWCNTs, 1-pyrenebutanoic acid, succinimidyl ester (PBASE), and anti-Staphylococcus aureus polyclonal antibodies (PABS) to the sensor platform. Error bars represent standard deviation $(n=3)$. Different letters $(\mathrm{a}-\mathrm{b})$ represent significant differences among the samples at $P<0.05$.

the sensor platform decreased. This result was in good agreement with the result of Wang and Musameh [23] in that the resistance values decreased as CNT concentrations increased. In the concentration ranges of 1 to $10 \mathrm{mg} / \mathrm{mL}$, the resistance values were very small. On the contrary, the resistance values at $0.02 \mathrm{mg} / \mathrm{mL}$ of SWCNTs were the highest among the tested concentrations; however, the resistance values were fluctuated with the washing steps. The fluctuation of resistance values might be attributed to the unstable immobilization of SWCNTs on the surface of the platform at $0.02 \mathrm{mg} / \mathrm{mL}-S W C N T$ concentration. The concentration of SWCNTs at $0.1 \mathrm{mg} / \mathrm{mL}$ exhibited a reasonable range of resistance value among the tested concentrations and more importantly maintained stable resistance values even after 5time washing steps. Therefore, the optimum concentration of SWCNTs for immobilization on the sensor surface was determined as $0.1 \mathrm{mg} / \mathrm{mL}$ in this study.

3.3. Immobilization of Antibody on SWCNT-Based Biosensor. The immobilization of antibody onto the CNT-immobilized sensor platform requires a linker between the CNT surface and antibody [21]. Many biological species can be noncovalently adsorbed on the CNT surfaces through hydrophobic, $\pi-\pi$ stacking, and/or electrostatic interactions [23]. 1Pyrenebutanoic acid, succinimidyl ester is widely used as a linker for CNTs [24]. The hydrophobic pyrenyl group of PBASE can be irreversibly adsorbed into the hydrophobic sidewall of a CNT through a $\pi-\pi$ stacking interaction [25].

As presented in Figure 3, the binding of PBASE with SWCNT surface did not significantly alter the resistance value of the SWCNT-based sensor platform. The succinimidyl ester groups on the other end of 1-pyrenebutanoic react with primary and secondary amines on the surface of the antibody for nucleophilic substitution in the presence of DMF solvent. Antibodies consist of two regions: the variable region and the constant region (stem of the Y). It is believed that the variable region of the antibody is defined for antigen binding, and the constant region reacts with effective cells or molecules [5]. 
TABLE 1: Resistance of SWCNT immobilized onto sensor platform.

\begin{tabular}{lcccccc}
\hline \multirow{2}{*}{ Concentration of SWCNTs $(\mathrm{mg} / \mathrm{mL})$} & \multirow{2}{*}{ No washing } & \multicolumn{4}{c}{ Resistance $(\mathrm{K} \Omega)$ after each washing } \\
& & 1st washing & 2nd washing & 3rd washing & 4th washing & 5th washing \\
\hline 0.02 & $233.4 \pm 38.8^{\mathrm{c}}$ & $328.5 \pm 57.4^{\mathrm{bc}}$ & $362.1 \pm 56.7^{\mathrm{bc}}$ & $406.8 \pm 90.2^{\mathrm{b}}$ & $340.1 \pm 62.1^{\mathrm{bc}}$ & $497.6 \pm 98.9^{\mathrm{a}}$ \\
0.1 & $4.30 \pm 0.82^{\mathrm{a}}$ & $4.70 \pm 0.97^{\mathrm{a}}$ & $4.92 \pm 1.07^{\mathrm{a}}$ & $4.86 \pm 1.00^{\mathrm{a}}$ & $4.86 \pm 1.03^{\mathrm{a}}$ & $4.77 \pm 1.27^{\mathrm{a}}$ \\
1 & $0.03 \pm 0.001^{\mathrm{a}}$ & $0.03 \pm 0.001^{\mathrm{a}}$ & $0.03 \pm 0.002^{\mathrm{a}}$ & $0.03 \pm 0.002^{\mathrm{a}}$ & $0.03 \pm 0.002^{\mathrm{a}}$ & $0.03 \pm 0.002^{\mathrm{a}}$ \\
10 & $0.02 \pm 0.004^{\mathrm{a}}$ & $0.03 \pm 0.008^{\mathrm{a}}$ & $0.06 \pm 0.018^{\mathrm{a}}$ & $0.07 \pm 0.02^{\mathrm{a}}$ & $0.07 \pm 0.021^{\mathrm{a}}$ & $0.07 \pm 0.023^{\mathrm{a}}$ \\
\hline
\end{tabular}

Different superscript letters $(\mathrm{a}-\mathrm{c})$ represent significant differences among the samples at $P<0.05$.

The antibody immobilization on the biosensor was achieved by reacting amino groups of the constant regions of the antibody with the succinimidyl ester of the linker to form covalent amide bonds.

The immobilization of antibody through PBASE significantly increased the resistance value of the biosensor platform $(P<0.05)$ (Figure 3). García-Aljaro et al. [21] reported that the increase in resistance of the biosensor, that is, the decrease in current, was due to the accumulation of negative charge from the antibodies. Other researchers reported similar results with functionalization of antibodies onto the CNTs by measuring electric current changes. Villamizar et al. [26] reported that the functionalization steps of anti-Salmonella antibodies decreased the electrical current of a typical CNT field effect transistor. The electrical current of the device decreased after the absorption of anti-Salmonella due to the charge transfer process in which the antibodies provide electrons to the CNTs [27]. These electrons are provided by the amid groups with amino acid residues, which results in a decrease in the electrical current and a shift in the threshold voltage of the CNTs.

3.4. Detection Response of SWCNT-Based Biosensor. In general, microbial metabolism usually results in an increase in both conductance and capacitance, causing a decrease in impedance [24]. In addition, the specific binding of bacteria with antibody can cause an increase in resistance and can be monitored by the detector such as potentiostats. The specific interaction between the microorganism and antibody hinders the current flow resulting in a significant increase in the resistance value step by step [25].

Figure 4 presents the detection responses of the SWCNTbased biosensor with $S$. aureus measured during each step of the fabrication of the biosensor. There were no significant differences in resistance values between the PBS buffer and bacterial culture, when the biosensor platforms were immobilized with SWCNTs only or SWCNTs and PBASE. However, there was a significant difference in resistance between PBS and bacterial culture after the sensor platform was immobilized with antibody $(P<0.05)$. The significant increase in the resistance value was speculated from the specific interaction between the antibody and $S$. aureus. This result indicated that $S$. aureus cells did not react with the SWCNT-based sensor platforms without the immobilization of antibodies. The result was in good agreement with the results of GarcíaAljaro et al. [21], in that the resistance values were increased as the steps of preparation of the biosensor were performed.

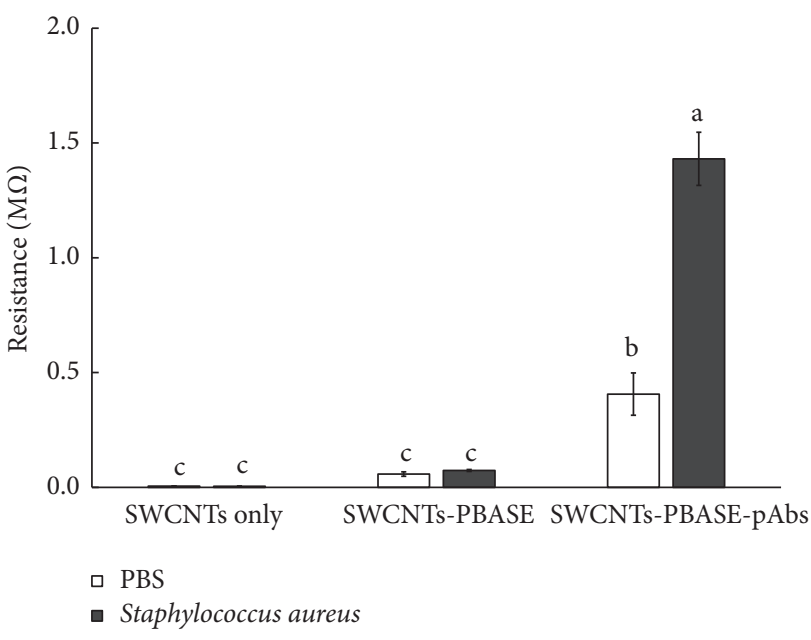

FIGURE 4: Resistance of SWCNT-based biosensor reacted with PBS buffer and Staphylococcus aureus culture. Error bars represent standard deviation $(n=3)$. Different letters $(\mathrm{a}-\mathrm{c})$ represent significant differences among the samples at $P<0.05$. PBASE and pAbs stand for 1-pyrenebutanoic acid, succinimidyl ester, and antiStaphylococcus aureus polyclonal antibodies, respectively.

3.5. SEM Images of S. aureus Cells Captured on the SWCNTBased Biosensor. The SEM microstructures of $S$. aureus on the SWCNT sensor platform are presented in Figure 4. The SWCNT-based biosensor that reacted with PBS buffer (control sensor) exhibited a typical thread-tangled shape of SWCNTs on the sensor platform surface under the SEM (Figure 5(a)). When the biosensor was applied with S. aureus cell culture, the SEM image revealed that the $S$. aureus was captured onto the surface of the SWCNT-based biosensor (Figure 5(b)). Villamizar et al. [26] also reported that SEM confirmed the number of Salmonella cells attached to the CNTs was proportional to the concentration of Salmonella in solution.

The SEM images demonstrated that the SWCNT-based biosensor could capture $S$. aureus cells, resulting in a significant increase in the resistance value of the biosensor as presented in Table 2. The biosensor immobilized with PBASE only was not able to capture $S$. aureus, exhibiting no significant resistance difference between the PBS buffer and bacterial cell culture. However, when the antibody was immobilized onto PBASE, the resistance difference was significantly different between the biosensors $(P<0.05)$. García-Aljaro et al. [21] reported the resistance increased after functionalizing 


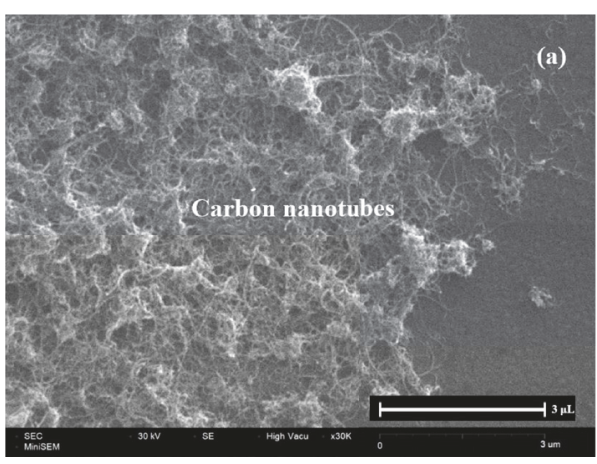

(a)

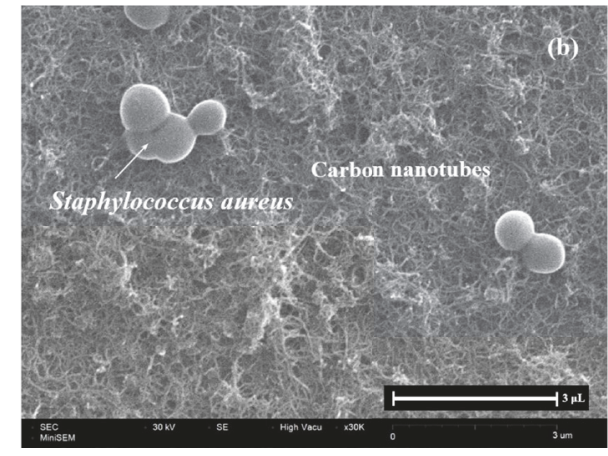

(b)

FIGURE 5: Scanning electron microscopic images of the detected Staphylococcus aureus by control sensor (a) and antibody-immobilized biosensor (b).

TABLE 2: Effect of immobilization steps on the resistance difference of SWCNT-based biosensor.

\begin{tabular}{lc}
\hline Immobilization step & Resistance difference $(\Delta R)$ \\
\hline PBASE only & $0.02 \pm 0.06$ \\
PBASE and pAbs & $9.78 \pm 0.47$
\end{tabular}

PBASE and pAbs stand for 1-pyrenebutanoic acid, succinimidyl ester and anti-Staphylococcus aureus polyclonal antibodies, respectively.

the biosensor with specific antibody for E. coli O157:H7 and concluded that the resistance increase was due to the specific binding of antibody with the bacteria on the CNT-based sensor.

3.6. Limit of Detection of SWCNT-Based Biosensor. The SWCNT-based biosensor immobilized with pAbs was exposed to increasing concentrations of $S$. aureus culture. As bacterial concentrations increased, the resistance differences also increased up to the bacterial concentration of $8 \log \mathrm{CFU} / \mathrm{mL}$. This is a typical characteristic of the functionalized CNT-based biosensor because the binding of the antibody with bacteria results in an increase in the resistance of the biosensor [21, 26]. The linear regression of the resistance differences with selected concentrations of bacterial culture is presented in Figure 6. The resistance difference of the SWCNT-based biosensor increased as the concentration of $S$. aureus cells increased, while the control sensor did not exhibit any difference in resistance. The regression coefficient $\left(R^{2}\right)$ of the SWCNT-based biosensor was determined to be 0.9634 . At the bacterial concentration of $4 \log \mathrm{CFU} / \mathrm{mL}$, the resistance difference began to differ significantly between the measurement biosensor and the control biosensor $(P<0.05)$. Therefore, the limit of detection (LOD) of SWCNT-based biosensor was determined to be the concentration of $10^{4} \mathrm{CFU} / \mathrm{mL}$. Villamizar et al. [26] reported that the biosensor response was a linear function of the logarithm of the bacterial concentrations between 3 and $7 \log \mathrm{CFU} / \mathrm{mL}\left(R^{2}=0.93\right)$. Pyun et al. [28] also reported that the limit of detection was determined to be $3.0 \times 10^{5} \mathrm{CFU} / \mathrm{mL}$,

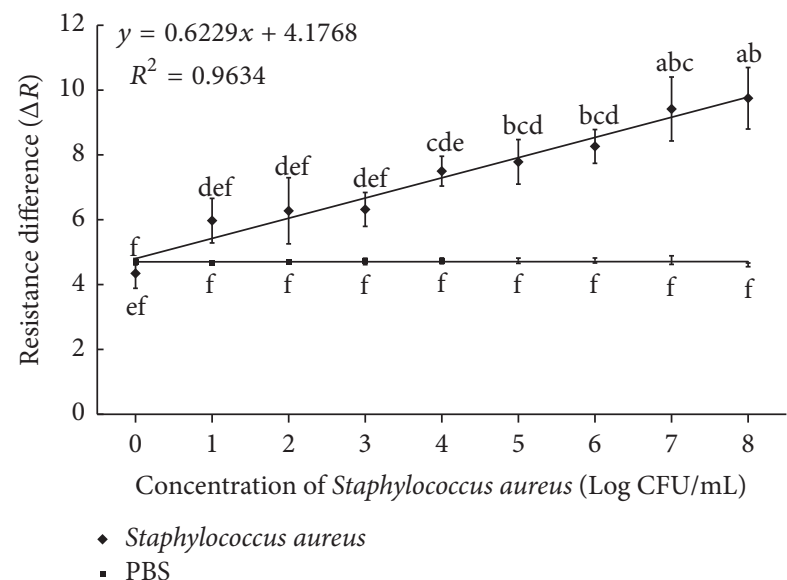

FIGURE 6: Resistance difference of SWCNT-based biosensor reacted with the selected concentration of Staphylococcus aureus from $10^{0}$ to $10^{8} \mathrm{CFU} / \mathrm{mL}$. Error bars represent standard deviation $(n=3)$. Different superscript letters $(\mathrm{a}-\mathrm{f})$ represent significant differences among the samples at $P<0.05$.

when a flexural plate wave transducer was used for the detection of $E$. coli.

The SWCNT-based biosensor developed in this study was not able to detect $S$. aureus less than $10^{4} \mathrm{CFU} / \mathrm{mL}$. Therefore, when a small amount of bacteria is present in food, it is necessary to perform an enrichment process to increase the bacterial number to a detectable level. A future study should be conducted in a way to increase the sensitivity of the biosensor by decreasing the LOD of the biosensor. Nonetheless, the SWCNT-based biosensor in this research may possess advantages compared with other techniques such as its miniaturized and simple structure, rapid detection less than $30 \mathrm{~min}$, and easy operation at room temperature.

\section{Conclusions}

This research demonstrated that the SWCNT-based biosensor could be useful for the detection of foodborne pathogens 
such as $S$. aureus. The indirect ELISA confirmed that the anti-S. aureus polyclonal antibodies were specific enough to bind to the target $S$. aureus cells among the tested bacteria. The gold electrodes fabricated on the silicon wafer were assembled with single-walled carbon nanotubes. The optimum concentration of assembled SWCNTs was determined to be $0.1 \mathrm{mg} / \mathrm{mL}$. 1-Pyrenebutanoic acid, succinimidyl ester was selected as a linker to bind between SWCNTs and pAbs. As PBASE and pAbs were immobilized on the biosensor platform, the resistance of the SWCNT-based biosensor increased. The captured S. aureus on the SWCNTbased biosensor was also viewed using a scanning electron microscope. As bacterial number increased, the resistance decreased. The SWCNT-based biosensors developed in this study was able to detect $S$. aureus with a concentration as low as $10^{4} \mathrm{CFU} / \mathrm{mL}$. Therefore, the limit of detection of the biosensor was determined to be $4 \log \mathrm{CFU} / \mathrm{mL}$.

\section{Additional Points}

Practical Applications. Recently, biosensor methods are receiving a great amount of attention because of the potential feasibility for detection of foodborne pathogens. A singlewalled carbon nanotube- (SWCNT-) based biosensor was developed and characterized to detect Staphylococcus aureus, one of the major food pathogens. The research demonstrated that the developed SWCNT-based biosensor could detect $S$. aureus specifically with a detection limit of $10^{4} \mathrm{CFU} / \mathrm{mL}$. This biosensor method was relatively simple, handy, and easy to operate. Therefore, the developed biosensor can be practically applied to detect food pathogens on-site in food industry.

\section{Disclosure}

Part of this research was presented as a poster at the Korean Society of Food Preservation (2015 Annual Meeting and Symposium).

\section{Conflicts of Interest}

The authors declare that there are no conflicts of interest regarding the publication of this paper. The support by Basic Science Research Program through the National Research Foundation (NRF) of Korea will not lead to any conflicts of interest regarding this publication.

\section{Acknowledgments}

This research was supported by Basic Science Research Program through the National Research Foundation (NRF) of Korea founded by the Ministry of Education (2014R1A1A2055619).

\section{References}

[1] J. Knox, A.-C. Uhlemann, and F. D. Lowy, "Staphylococcus aureus infections: transmission within households and the community," Trends in Microbiology, vol. 23, no. 7, pp. 437-444, 2015.
[2] Korean Ministry of Food and Drug Safety, 2017, http://www .foodsafetykorea.go.kr/portal/healthyfoodlife/foodPoisoningStat.do?menu_no=519\&amp;menu_grp=MENU_GRP02.

[3] Y. L. Loir, F. Baron, and M. Gautier, "Staphylococcus aureus and food poisoning," Genetics and Molecular Research, vol. 2, no. 1, pp. 63-76, 2003.

[4] J. D. Oliver, "Recent findings on the viable but nonculturable state in pathogenic bacteria," FEMS Microbiology Reviews, vol. 34, no. 4, pp. 415-425, 2010.

[5] N. Wang, M. He, and H.-C. Shi, "Novel indirect enzyme-linked immunosorbent assay (ELISA) method to detect Total E. coli in water environment," Analytica Chimica Acta, vol. 590, no. 2, pp. 224-231, 2007.

[6] M. Chandra, P. Cheng, G. Rondeau, S. Porwollik, and M. McClelland, "A single step multiplex PCR for identification of six diarrheagenic E. coli pathotypes and Salmonella," International Journal of Medical Microbiology, vol. 303, no. 4, pp. 210 216, 2013.

[7] S.-M. Wu, J. Chen, X.-X. Ai, and Z.-Y. Yan, "Detection of Escherichia coli in drugs with antibody conjugated quantum dots as immunofluorescence probes," Journal of Pharmaceutical and Biomedical Analysis, vol. 78-79, pp. 9-13, 2013.

[8] L. N. Cella, W. Chen, N. V. Myung, and A. Mulchandani, "Single-walled carbon nanotube-based chemiresistive affinity biosensors for small molecules: ultrasensitive glucose detection," Journal of the American Chemical Society, vol. 132, no. 14, pp. 5024-5026, 2010.

[9] H. Dai, "Carbon nanotubes: synthesis, integration, and properties," Accounts of Chemical Research, vol. 35, no. 12, pp. 10351044, 2002.

[10] A. Merkoçi, M. Pumera, X. Llopis, B. Pérez, M. Del Valle, and S. Alegret, "New materials for electrochemical sensing VI: carbon nanotubes," TrAC Trends in Analytical Chemistry, vol. 24, no. 9, pp. 826-838, 2005.

[11] P. Avouris and J. Chen, "Nanotube electronics and optoelectronics," Materials Today, vol. 9, no. 10, pp. 46-54, 2006.

[12] W. Kusnezow and J. D. Hoheisel, "Solid supports for microarray immunoassays," Journal of Molecular Recognition, vol. 16, no. 4, pp. 165-176, 2003.

[13] F. Khan, M. He, and M. J. Taussig, "Double-hexahistidine tag with high-affinity binding for protein immobilization, purification, and detection on Ni-nitrilotriacetic acid surfaces," Analytical Chemistry, vol. 78, no. 9, pp. 3072-3079, 2006.

[14] E. Katz, I. Willner, and A. B. Kotlyar, "A non-compartmentalized glucose-O2 biofuel cell by bioengineered electrode surfaces," Journal of Electroanalytical Chemistry, vol. 479, no. 2, pp. 64-68, 1999.

[15] A. Ramanavicius, A. Kausaite, and A. Ramanaviciene, "Biofuel cell based on direct bioelectrocatalysis," Biosensors and Bioelectronics, vol. 20, no. 10, pp. 1962-1967, 2005.

[16] J. Y. Lee, H. Y. Shin, J. H. Lee et al., "A novel enzyme-immobilization method for a biofuel cell," Journal of Molecular Catalysis B: Enzymatic, vol. 59, no. 4, pp. 274-278, 2009.

[17] O. Lazcka, F. J. D. Campo, and F. X. Muñoz, "Pathogen detection: a perspective of traditional methods and biosensors," Biosensors and Bioelectronics, vol. 22, no. 7, pp. 1205-1217, 2007.

[18] M.-K. Park, J. W. Park, and J.-H. Oh, "Optimization and application of a dithiobis-succinimidyl propionate-modified immunosensor platform to detect Listeria monocytogenes in chicken skin," Sensors and Actuators B: Chemical, vol. 171-172, pp. 323-331, 2012. 
[19] S. Zhang, T.-S. Huang, R. Bridgman, and J. Weese, "Development of characterization of monoclonal and polyclonal antibodies against Salmonella enterica Typhimurium for biosensor detection," Journal of Food Science, vol. 71, no. 3, pp. M100M104, 2006.

[20] J. Y. Lee, H. Y. Shin, S. W. Kang, C. Park, and S. W. Kim, "Improvement of electrical properties via glucose oxidaseimmobilization by actively turning over glucose for an enzymebased biofuel cell modified with DNA-wrapped single walled nanotubes," Biosensors and Bioelectronics, vol. 26, no. 5, pp. 2685-2688, 2011.

[21] C. García-Aljaro, L. N. Cella, D. J. Shirale et al., "Carbon nanotubes-based chemiresistive biosensors for detection of microorganisms," Biosensors and Bioelectronics, vol. 26, no. 4, pp. 1437-1441, 2010.

[22] X. Zhou, J.-Y. Park, S. Huang, J. Liu, and P. L. McEuen, "Band structure, phonon scattering, and the performance limit of single-walled carbon nanotube transistors," Physical Review Letters, vol. 95, no. 14, Article ID 146805, 2005.

[23] J. Wang and M. Musameh, "Carbon nanotube/Teflon composite electrochemical sensors and biosensors," Analytical Chemistry, vol. 75, no. 9, pp. 2075-2079, 2003.

[24] D. Ivnitski, I. Abdel-Hamid, P. Atanasov, and E. Wilkins, "Biosensors for detection of pathogenic bacteria," Biosensors and Bioelectronics, vol. 14, no. 7, pp. 599-624, 1999.

[25] P. He and L. Dai, "Carbon nanotube biosensors," in Biological and Biomedical Nanotechnology, A. P. Lee and L. J. Lee, Eds., pp. 171-201, Springer, New York, NY, USA, 2006.

[26] R. A. Villamizar, A. Maroto, F. X. Rius, I. Inza, and M. J. Figueras, "Fast detection of Salmonella Infantis with carbon nanotube field effect transistors," Biosensors and Bioelectronics, vol. 24, no. 2, pp. 279-283, 2008.

[27] G. Gruner, "Carbon nanotube transistors for biosensing applications," Analytical and Bioanalytical Chemistry, vol. 384, no. 2, pp. 322-335, 2006.

[28] J. C. Pyun, H. Beutel, J.-U. Meyer, and H. H. Ruf, "Development of a biosensor for E. coli based on a flexural plate wave (FPW) transducer," Biosensors and Bioelectronics, vol. 13, no. 7-8, pp. 839-845, 1998. 

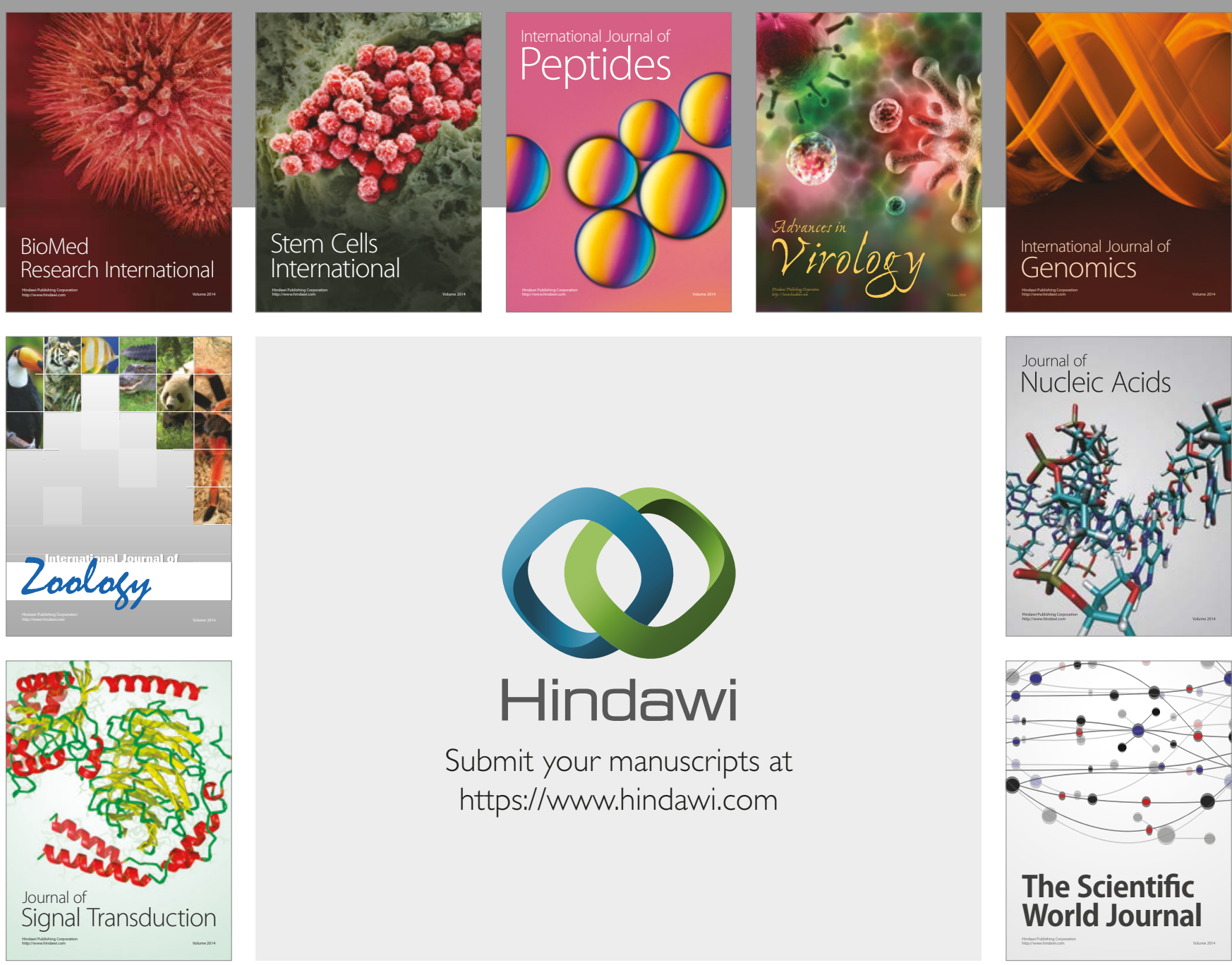

Submit your manuscripts at

https://www.hindawi.com
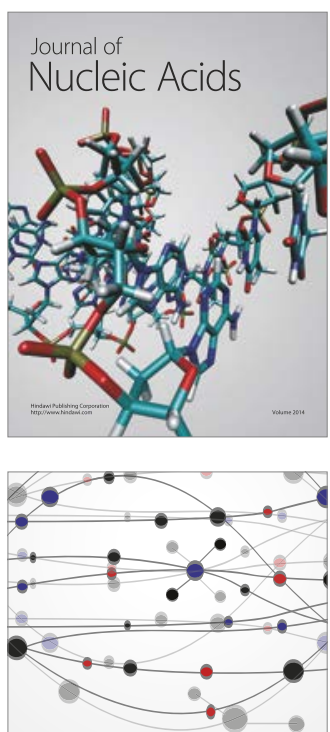

The Scientific World Journal

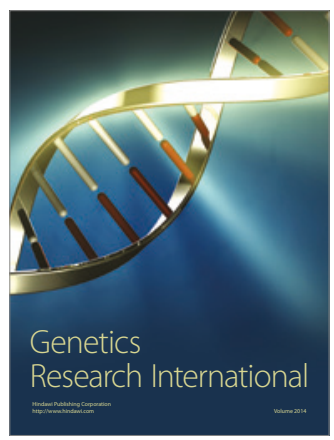

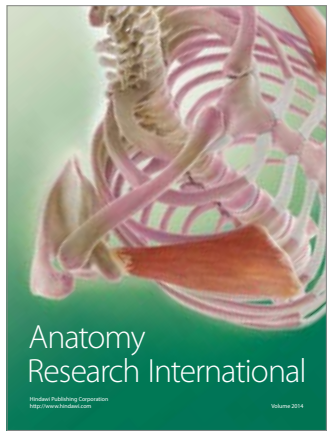

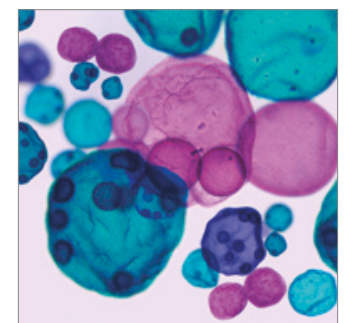

International Journal of Microbiology
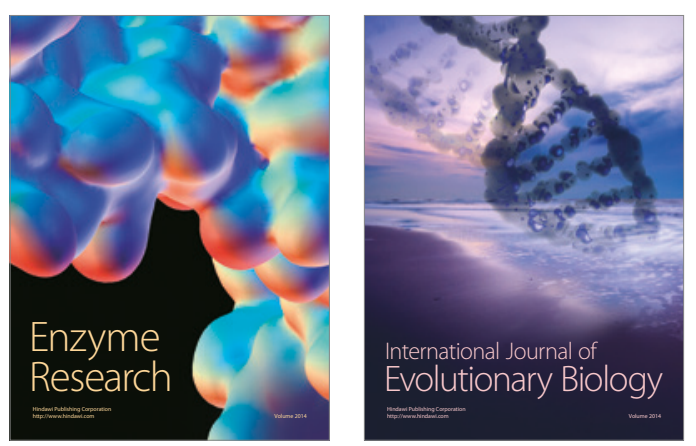
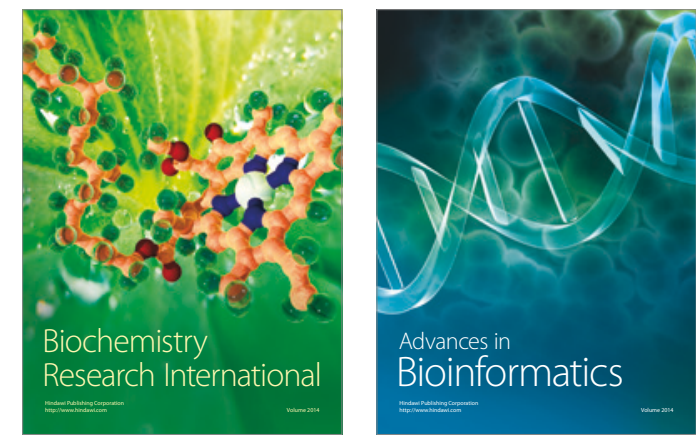

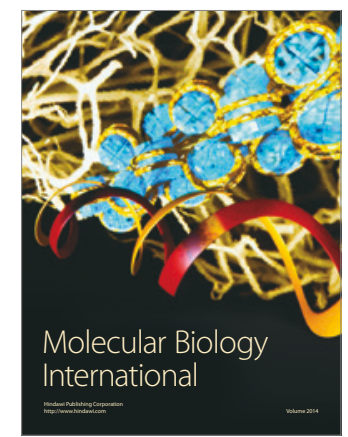

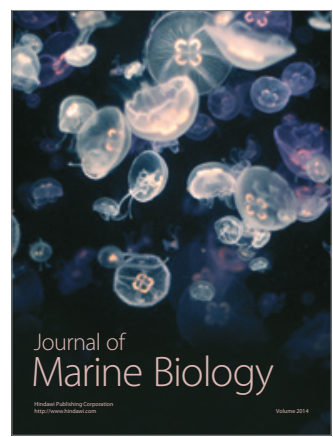

